www.nature.com/pj

\title{
A versatile photochemical procedure to introduce a photoreactive molecular layer onto a polyimide film for liquid crystal alignment
}

\author{
Akira Sasaki $^{1}$, Hozumi Aoshima ${ }^{1}$, Shusaku Nagano ${ }^{2,3}$ and Takahiro Seki ${ }^{1}$ \\ A polyimide film containing a cholesteryl moiety spin-coated on a solid substrate is treated with vacuum ultraviolet light from \\ a $\mathrm{Xe}_{2}{ }^{*}$ excimer lamp at $20 \mathrm{~Pa}$. This procedure generates oxidized chemically functional groups, such as hydroxyl groups, \\ selectively on the top-most surface without changing the nature of the bulk polymer. Onto the chemically functionalized \\ surface, photofunctional units such as azobenzene and cinnamate units are attached at a monolayer or sub-monolayer level. \\ When nematic liquid crystal (LC) cells are fabricated, such photoresponsive surfaces work as effective 'command surfaces' \\ for photoswitching the zenithal LC orientation and photoaligment layers to achieve the homogeneous azimuthal orientation \\ applicable for vertical alignment mode LC devices. The proposed photochemical treatment provides a simple but versatile \\ alternative process for the preparation of surface molecular films comparable to self-assembled monolayers or Langmuir- \\ Blodgett films.
}

Polymer Journal (2012) 44, 639-645; doi:10.1038/pj.2012.45; published online 28 March 2012

Keywords: azobenzene; cinnamate; liquid crystal cell; photoalignment; polyimide film; surface modification; vacuum ultraviolet light

\section{INTRODUCTION}

In liquid crystal (LC) display devices, the aligned layers of LCs are commonly used, and the alignment is typically determined by an anisotropic solid surface. Owing to the long-range orientational interaction, the preferred alignment of an LC determined by this surface extends into the LC bulk on a macroscopic scale. This photoalignment effect was first described in 1988 by Ichimura et al. ${ }^{1}$ (Figure 1a). Light irradiation of the aligning substrate covered with azobenzene $(\mathrm{Az})$ monolayers resulted in homeotropic-to-planar reorientation of the LC due to the trans-cis isomerization of Az. This process is reversible by varying the wavelength of light used. After this discovery, Gibbons et al. ${ }^{2}$ and Schadt et al. ${ }^{3}$ (Figure $1 \mathrm{~b}$ ) demonstrated photoalignment in an azimuthal plane of the aligning substrate using linearly polarized light. The former and the latter cases utilized an Az dye and a cinnamate unit, respectively. Photoalignment technology provides non-contact and effective control of basic anchoring parameters, namely an easy orientation axis, pre-tilt angle and anchoring energy. ${ }^{4-6}$

To control the alignment of nematic LCs, the occurrence of the photoreaction throughout the thickness of a coated polymer film is not necessary; the introduction of a photoreactive layer on the top-most surface is sufficient because the molecular orientation at the contacting substrate surface boundary critically affects the nematic LC alignment. The introduction of such a layer is thus far achieved by setting a self-assembled monolayer ${ }^{1,7,8}$ or a Langmuir-Blodgett monolayer. ${ }^{9,10}$ In this paper, we propose a new alternative and versatile procedure using a photochemical treatment on a polymer surface to set a photoaligning molecular layer.

Surface modifications of polymer surfaces have been widely performed using plasma, ${ }^{10-15}$ corona discharge, ${ }^{16,17}$ ultraviolet (UV) light, ${ }^{17,18}$ ion beams ${ }^{19}$ or bombardment, ${ }^{20-22} \gamma$-radiation, ${ }^{23-25}$ and chemical solutions ${ }^{26-28}$ to modify macroscopic surface properties, such as wettability, friction, adhesion, biocompatibility, molecular recognition and LC alignment. In most cases, the formation of oxygen-containing functional groups, such as $-\mathrm{OH}$ and $-\mathrm{COOH}$, occurs on the polymer surfaces. The use of such functional groups to attach a photoreactive group covalently is anticipated.

In the present approach, we attempted to use an inexpensive light source of vacuum ultraviolet (VUV, $172 \mathrm{~nm}$ ) light of a $\mathrm{Xe}_{2}{ }^{*}$ excimer lamp ${ }^{29,30}$ for the surface modification. Previous studies have discussed the mechanism for the photodecomposition of organic compounds

\footnotetext{
${ }^{1}$ Department of Molecular Design and Engineering, Graduate School of Engineering, Nagoya University, Nagoya, Japan; ${ }^{2}$ Venture Business Laboratory, Graduate School of Engineering, Nagoya University, Nagoya, Japan and ${ }^{3}$ Precursory Research for Embryonic Science and Technology (PRESTO), Japan Science and Technology Agency (JST), Kawaguchi, Japan

Correspondence: Professor S Nagano, Venture Business Laboratory, Graduate School of Engineering, Nagoya University, Chikusa-ku, Nagoya, Japan.

E-mail: snagano@apchem.nagoya-u.ac.jp

or Professor T Seki, Department of Molecular Design and Engineering, Graduate School of Engineering, Nagoya University, Chikusa-ku, Nagoya 464-8603, Japan.

E-mail: tseki@apchem.nagoya-u.ac.jp
}

Received 13 January 2012; revised 31 January 2012; accepted 12 February 2012; published online 28 March 2012 
and materials by VUV light in the presence of oxygen. ${ }^{31-33}$ The VUV treatment can be expected to be applicable to many types of polymer materials. Among them, we have selected a polyimide film because of its practical importance in LC alignment due to its excellent bulk properties, including its chemical resistance, low dielectric property and mechanical robustness. The advantage of this method is that only the top-most surface of the polymer film is modified without changing its bulk characteristic features, as stated above. The attachment of two typical photoreactive units, Az (photoisomerization) and cinnamoyl (photodimerization) on the surface is attempted. The scheme of a series of processes and the chemical structure of the materials are depicted in Figure 2. A cholesteryl

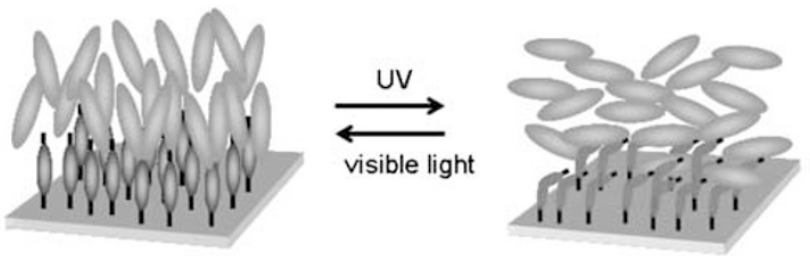

Zenithal (out-of-plane) switching by an azobenzene monolayer

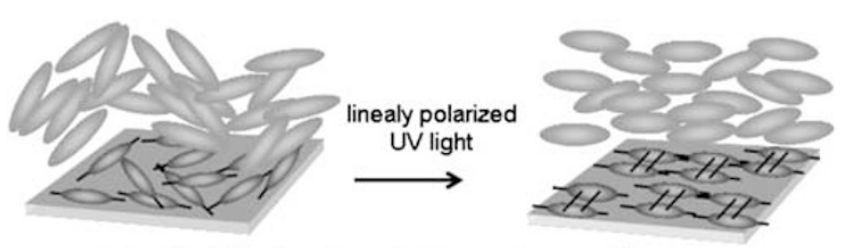

Azimuthal (in-plane) control by a cinnamoyl layer

Figure 1 Schematic illustrations of surface-mediated orientational control of nematic LC molecules using light irradiation. side moiety was introduced in the polyimide structure to achieve vertical alignment mode switching in response to an electric field. ${ }^{34,35}$

\section{EXPERIMENTAL PROCUDURE}

\section{Materials}

5-(Dimethylamino)naphthalene-1-sulfonyl chloride (dansyl chloride, fluorescent labeling reagent for hydroxyl group), 3-bromomethyl-7-methoxy-1,4-bonzoxazine-2-one (Br-MB, fluorescent labeling reagent for carboxyl acid group) cinnamoyl chloride, 4'-pentyl-4-cyanobiphenyl (5CB), and 5-(2,5-dioxotetrahydrofuryl)-3-methyl-3-cyclohexene-1,2-dicarboxylic anhydride were purchased from Tokyo Chemical Industry Co. Ltd. (Tokyo, Japan) and were used according to the manufacturer's protocol. 11-(4-((4-Pentylphenyl)azo)phenoxy)undecanoyl chloride $(5 \mathrm{Az} 10 \mathrm{COCl})$ was synthesized using an approach described previously. ${ }^{9}$ A nematic LC of DON-103 and dichroic dye (LCD-118) were acquired from DIC (Tokyo, Japan) and Nippon Kayaku (Tokyo, Japan) respectively.

Cholesteryl 3.5-dinitrobenzoate. This compound was synthesized according to the method described in the literature ${ }^{35}$ at a yield of $74 \%$. Melting point: $197-204{ }^{\circ} \mathrm{C},{ }^{1} \mathrm{H}$-nuclear magnetic resonance ( $\delta$ (p.p.m.), $270 \mathrm{MHz}, \mathrm{CDCl}_{3}$ ): 0.6-2.7 (43H, m, CH), 4.8-5.3 (1H, m, $-\mathrm{O}-\mathrm{CH}-)$, 5.4-5.5 (1H, m, C=CH), $9.13(2 \mathrm{H}, \mathrm{d}, J=1.9 \mathrm{~Hz}, \mathrm{Ar}-\mathrm{H}), 9.20(1 \mathrm{H}, \mathrm{t}, J=1.9 \mathrm{~Hz}, \mathrm{Ar}-\mathrm{H})$.

Cholesteryl 3.5-diaminobenzoate. Cholesteryl 3,5-dinitrobenzoate $(4.0 \mathrm{~g}$ $(6.8 \mathrm{mmol}))$ was dissolved in a dry mixture of tetrahydrofuran $(160 \mathrm{ml})$ and ethanol $(40 \mathrm{ml})$ and fed into a $300-\mathrm{ml}$ three-necked flask. Into this solution, a $\mathrm{Pt} /$ carbon (Pd 5\%) catalyst was added, heated to $60^{\circ} \mathrm{C}$ and stirred for $10 \mathrm{~min}$. Next, hydrazine monohydrate $(4.0 \mathrm{~g}(80 \mathrm{mmol}))$ was slowly added. After stirring for an additional $12 \mathrm{~h}$ at $60^{\circ} \mathrm{C}$, the resultant mixture was cooled to room temperature, the catalyst was filtered off and the filtered solution was evaporated. The resulting solid was recrystallized from ethyl acetate to obtain a white powder at a yield of $60 \%(2.2 \mathrm{~g})$. Melting point: $187-192{ }^{\circ} \mathrm{C},{ }^{1} \mathrm{H}$-nuclear magnetic resonance $\left(\delta\right.$ (p.p.m.), $\left.270 \mathrm{MHz}, \mathrm{CDCl}_{3}\right): 0.6-2.7(43 \mathrm{H}, \mathrm{m}, \mathrm{CH})$,

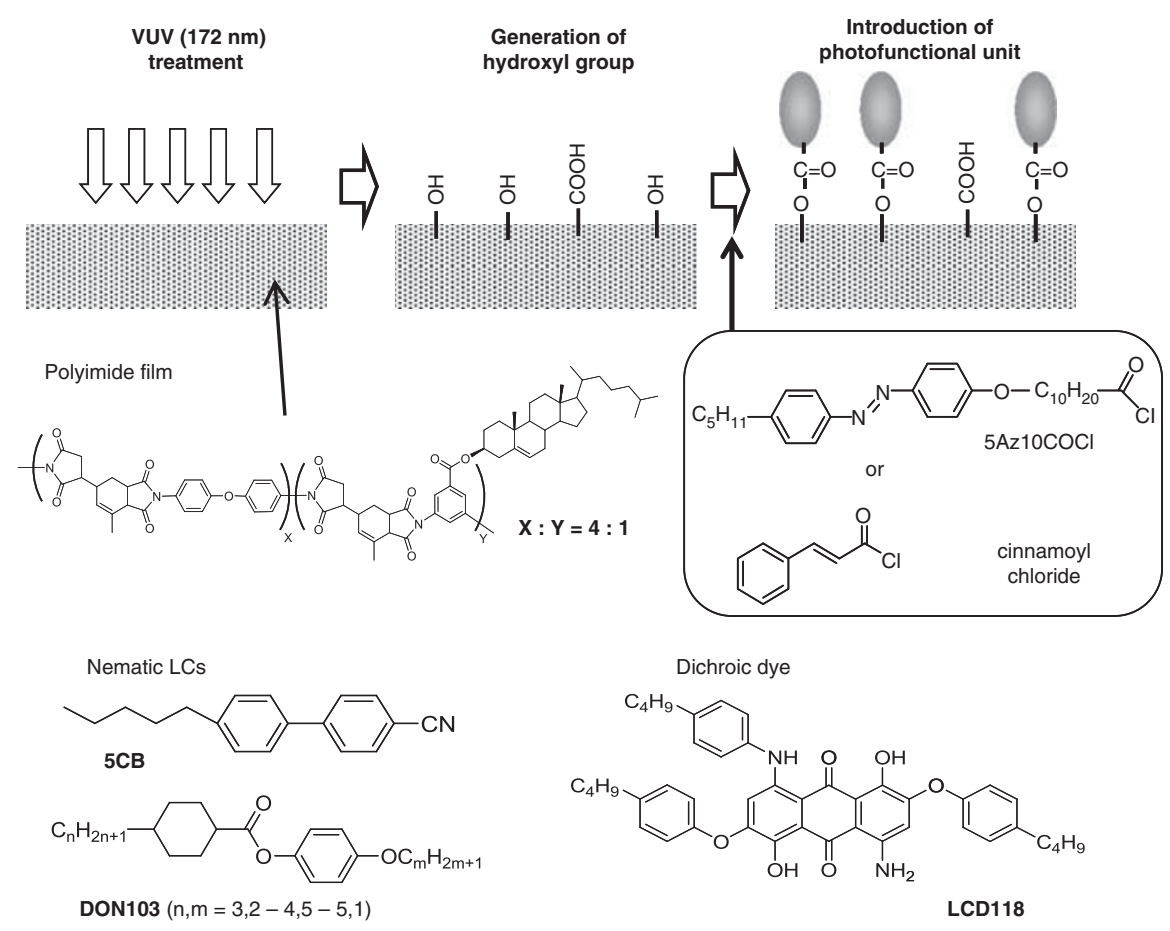

Figure 2 Proposed VUV light irradiation procedure to introduce photoresponsive units on a polyimide surface, and an illustration of the materials used in this study. 
3.6-3.8 $\left(4 \mathrm{H}, \mathrm{s}, \mathrm{NH}_{2}\right), 4.7-5.2(1 \mathrm{H}, \mathrm{m},-\mathrm{O}-\mathrm{CH}-), 5.4-5.5(1 \mathrm{H}, \mathrm{m}, \mathrm{C}=\mathrm{CH})$, $6.17(1 \mathrm{H}, \mathrm{t}, J=2.2 \mathrm{~Hz}, \mathrm{Ar}-\mathrm{H}), 6.78(2 \mathrm{H}, \mathrm{d}, J=2.2 \mathrm{~Hz}, \mathrm{Ar}-\mathrm{H})$.

Polyamide acid. The precursor polymer for polyimide was synthesized as follows. Bis $(4$-aminophenyl $)$ ether $(1.6 \mathrm{~g}(8.0 \mathrm{mmol}))$ and cholesteryl 3,5-diaminobenzoate $(1.04 \mathrm{~g}(2.00 \mathrm{mmol}))$ were dissolved in dry $\mathrm{N}$-methylpyrrolidone and placed in a 100-ml-three-necked flask purged with nitrogen gas. Into this solution, 5-(2,5-dioxotetrahydrofuryl)-3-methyl-3-cyclohexene1,2-dicarboxylic anhydride was carefully added and heated to $60^{\circ} \mathrm{C}$ for $48 \mathrm{~h}$. After cooling to room temperature, additional $\mathrm{N}$-methylpyrrolidone was added to obtain a 2.5 -wt $\%$ stock solution.

\section{Methods}

The polyamide acid solution $(2.5 \mathrm{wt} \%)$ in $N$-methylpyrrolidone was spincoated on a quartz substrate or an indium-tin-oxide electrode at 2000 r.p.m. for $60 \mathrm{~s}$. The coated film was pre-baked at $90{ }^{\circ} \mathrm{C}$ for $10 \mathrm{~min}$ and cured at $180^{\circ} \mathrm{C}$ for $1 \mathrm{~h}$ to obtain a polyimide film. The polymer film was $\sim 50 \mathrm{~nm}$ thick.

The sample was placed in a vacuum chamber evacuated by a rotary pump. The residual pressure in the chamber was controlled at $20 \mathrm{~Pa}$ by introducing some air through a variable leak valve. The polyimide film was exposed to $172 \mathrm{~nm}$ VUV light radiated from a $\mathrm{Xe}_{2}{ }^{*}$ excimer lamp (Ushio Inc., Tokyo, Japan, UER20-172V, $\lambda=172 \mathrm{~nm}, 10 \mathrm{~mW} \mathrm{~cm}^{-2}$ ) for $60 \mathrm{~s}$ with a distance of $\sim 20 \mathrm{~mm}$ between the lamp window and the sample surface.

Surface modification using dansyl chloride (reactive with the hydroxyl group) was achieved as follows. The film was exposed to VUV in theaforementioned manner and immersed into anhydrous tetrahydrofuran (THF) (30 ml) containing dansyl chloride $(30 \mathrm{mg})$, 4-dimethylaminopyridine $(1 \mathrm{mg})$ and triethylamine $(150 \mu \mathrm{l})$. The film was immersed in the solution at $25^{\circ} \mathrm{C}$ for $2 \mathrm{~h}$. The resulting sample was washed with THF and dried. Surface modification with Br-MB (reactive with carboxylic acid) was performed in the following manner. Potassium carbonate was dissolved in ethanol $(25 \mathrm{ml})$ to a saturated concentration. The VUV-patterned substrate was then immersed into this solution. After $30 \mathrm{~min}, \mathrm{Br}-\mathrm{MB}(30 \mathrm{mg})$ was added and stirred at room temperature for $2 \mathrm{~h}$. The resulting sample was washed with ethanol and then dried.

The surface modification using a photoresponsive molecule $(5 \mathrm{Az} 10 \mathrm{COCl}$ or cinnamoyl chloride) on the oxidized polyimide surface was performed using a similar approach as that used for the dansyl chloride. Photoirradiation to an Az-modified surface was performed using a 200-W mercury xenon light (UVF204S, San-Ei Electric Co., Osaka, Japan) through an appropriate combination of optical filters. The cinnamoyl-modified surface was exposed to linearly polarized UV light (low-power laser (LPL), $400 \mathrm{~mJ} \mathrm{~cm}^{-2}$ at $313 \mathrm{~nm}$ ) with the same lamp through a glass slide that acted as a cutoff filter for light with a wavelength below $300 \mathrm{~nm}$.

After exposure to UV light, LC cells with a 4- $\mu \mathrm{m}$ gap were assembled using the chemically modified polyimide films. A nematic LC of 5CB or DON-103 was injected between the cell gap using a capillary method above the isotropidization temperature, and the injection hole was then sealed with a photo-curable epoxy resin.

The voltage-transmittance $(V-T)$ characteristics of the vertical aligned-LC cells were evaluated using a homemade setup. An alternate current was applied at $500 \mathrm{~Hz}$.

\section{Measurements}

The water-droplet contact angles were measured using a CA-XP (Kyowa Interface, Saitama, Japan) instrument. The measurement was achieved after a droplet of $1 \mu \mathrm{l}$ was gently placed. The contact angle values were obtained from the average of five measurements.

Atomic force microscopy measurements were taken using a Nanopics 2100 (Seiko Instruments Inc., Chiba, Japan) to evaluate the polyimide film thickness and surface roughness. The film was scratched with a spatula, and the film height was measured.

UV-vis absorption spectra were taken on an Agilent 8453 spectrophotometer (Agilent Technologies, Santa Clara, CA, USA).
An OLYMPUS BX51 microscope (Olympus, Tokyo, Japan) was used for fluorescence microscopic observation of the patterned dansyl layer and polarized optical microscopic evaluations (using crossed polarizers) of the LC texture.

X-ray photoelectron spectroscopy was performed using a JPS-9000 MC (JEOL, Tokyo, Japan). X-rays $\left(\mathrm{Al} \mathrm{K}_{\alpha}\right.$ ) at $10 \mathrm{kV}$ were used for the measurements. Take-off angles of 5 and $90^{\circ}$ were used, and spectra with a resolution of $0.05 \mathrm{eV}$ were obtained 16 times.

\section{RESULTS AND DISCUSSION}

\section{Characterizations of a VUV-treated polyimide surface}

The film thickness before and after VUV was virtually unchanged (from 50.6 to $50.1 \mathrm{~nm}$ ). The surface roughness (the average rootmean-square) also remained essentially constant (root-mean-square from 1.63 to $1.40 \mathrm{~nm}$ ). Thus, VUV irradiation leads to no changes in the morphological features of the polymer film.

In contrast, the chemical characteristics of the surface were significantly altered. Before irradiation with VUV light, the contact angle with a water droplet was evaluated to be $101^{\circ}$ on the polyimide film under investigation. Using irradiation with VUV light at $20 \mathrm{~Pa}$, the contact angle rapidly decreased. After $60 \mathrm{~s}$ of irradiation, the contact angle was reduced to $87^{\circ}$ and remained almost constant at $75^{\circ}$ after further irradiation for $600 \mathrm{~s}$. The decrease in the contact angle of water clearly indicates the generation of polar groups on the surface.

$\mathrm{X}$-ray photoelectron spectroscopy spectra were measured for the $\mathrm{C}_{1 \mathrm{~s}}$ and $\mathrm{O}_{1 \mathrm{~s}}$ regions. In this setup, measurements at take-off angles of 5 and $90^{\circ}$ provided information for depths of $\sim 0.8 \mathrm{~nm}$ and $9.0 \mathrm{~nm}$, respectively. ${ }^{36}$ In Figures $3 \mathrm{a}$ and $\mathrm{b}$, the spectra taken at $5^{\circ}$ are displayed. In both the $\mathrm{C}_{1 \mathrm{~s}}$ (a) and $\mathrm{O}_{1 \mathrm{~s}}$ (b) regions, signals from the $\mathrm{C}-\mathrm{O}$ and $\mathrm{C}=\mathrm{O}$ bonds were enhanced with concomitant decreases in the $\mathrm{C}-\mathrm{H}$ and $\mathrm{C}-\mathrm{C}$ signals. These results suggest that oxidation occurred on the surface. The intensity (area) ratios of $\mathrm{O}_{1 \mathrm{~s}} / \mathrm{C}_{1 \mathrm{~s}}$ are shown in Figure 3c. The $\mathrm{O}_{1 \mathrm{~s}} / \mathrm{C}_{1 \mathrm{~s}}$ ratio increased as the irradiation time increased. At a take-off angle of $5^{\circ}$, the ratio was enhanced from 0.44 to 0.66 after VUV light treatment for $300 \mathrm{~s}$. The increase in the ratio was more prominent for the take-off angle of $5^{\circ}$, indicating that oxidation selectively occurred at the surface region of the polyimide film within $\sim 0.9 \mathrm{~nm}$. In Figure 3d, transmission Fourier transform infrared spectroscopy spectra of the polyimide films before and after VUV light irradiation are compared. From this measurement, information about the chemical bonds for all film thickness regions was obtained. No change was observed between the two spectra, indicating that the oxidation proceeded only on the polyimide film surface without changing the chemical nature of the bulk.

To confirm the reactivity of the oxidized surface, a fluorescent labeling reagent of dansyl chloride was reacted with the surface after patterned VUV irradiation (figures of ' $\mathrm{A}$ ' and ' $\mathrm{S}$ '). In Figure 4, a fluorescence microscopic image of the polyimide film after this procedure is presented. The fluorescence from the attached dansyl sulfonate is clearly observed in the irradiated areas of the photomask. As acid chloride was reacted with the surface, hydroxyl groups were generated at the polyimide film surface. We also attempted to label the VUV-exposed surface with $\mathrm{Br}-\mathrm{MB}$, a fluorescent labeling reagent for the carboxyl acid group. In this case, the contrast of the pattern was unclear. Therefore, the major oxygen-containing functional group is composed primarily of a hydroxyl group.

\section{Attachment of an Az unit and the photoresponse of an LC cell} For the standard VUV irradiation condition, we adopted an irradiation of $60 \mathrm{~s}$ to obtain good reproducibility of the chemical modification. An $\mathrm{Az}$ derivative (5Az10COCl) was reacted with the VUVtreated surface as performed for dansyl chloride. The UV-visible 

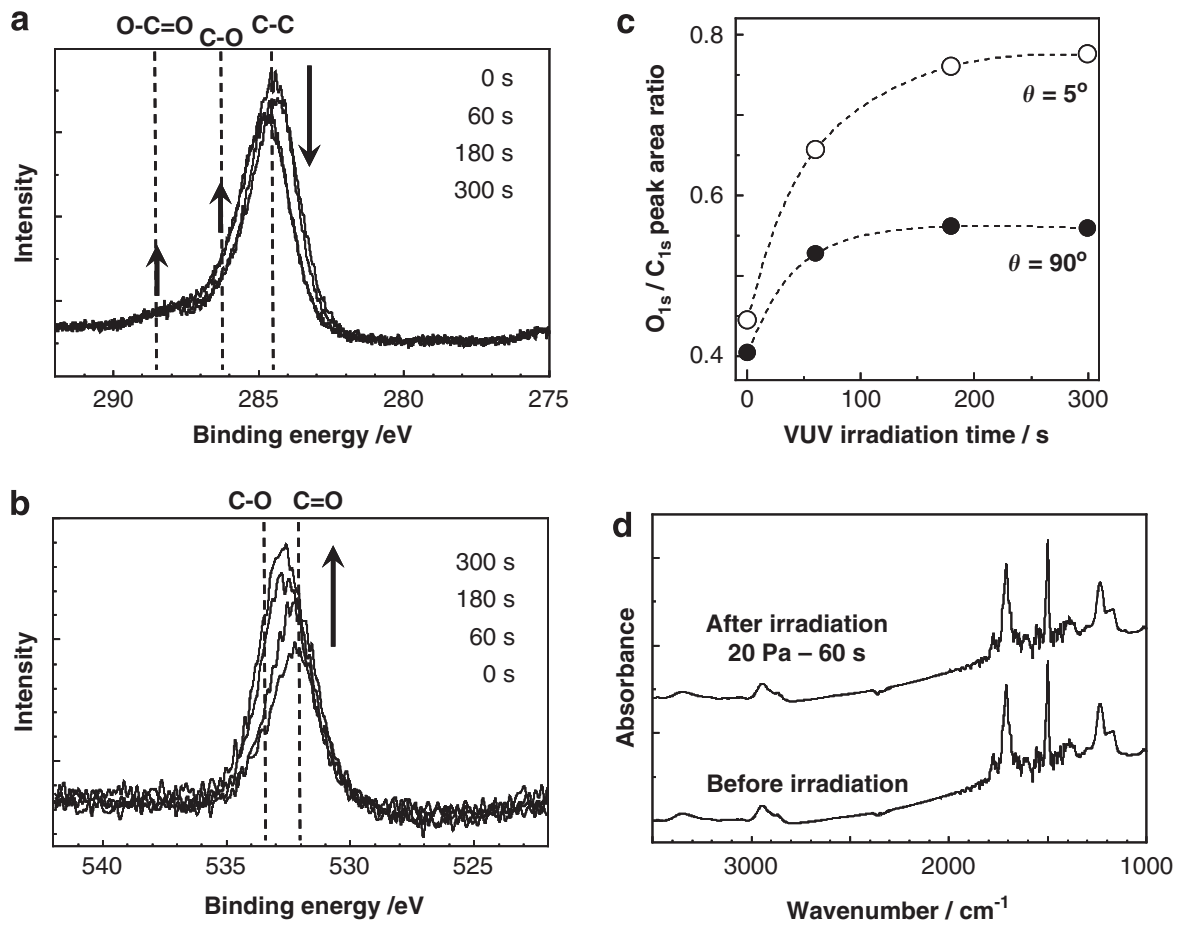

Figure 3 Characterization of the VUV-irradiated polyimide film. Changes in the X-ray photoelectron spectroscopy (a) $\mathrm{C}_{1 \mathrm{~s}}$ and (b) $\mathrm{O}_{1 \mathrm{~s}}$ narrow spectrum of the polyimide thin films at a take-off angle of $5^{\circ}$ before and after VUV light irradiation. (c) Changes in the area ratio of $\mathrm{O}_{1 \mathrm{~s}} / \mathrm{C}_{1 \mathrm{~s}}$ with VUV irradiation time taken at different take-off angles. (d) Transmission Fourier transform infrared spectroscopy spectra of the polyimide thin film before and after VUV light irradiation.

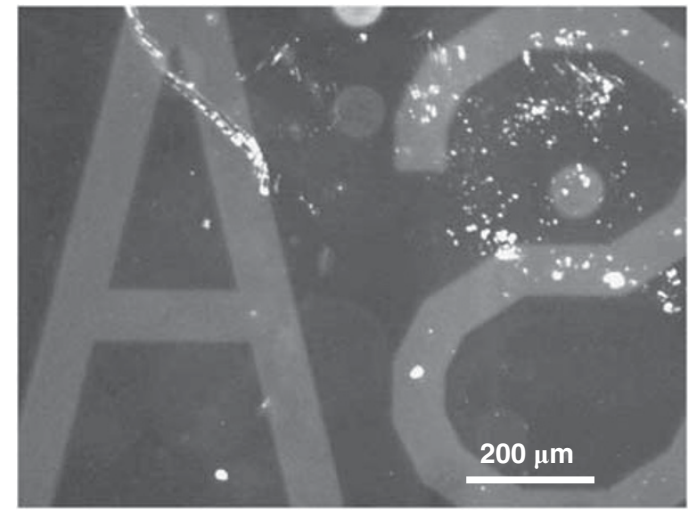

Figure 4 Fluorescence microscopic image of a polyimide film after patterned irradiation with VUV light followed by a reaction with dansyl chloride. The bright are as correspond to the VUV-irradiated parts.

absorption spectra are presented in Figure 5. After the attachment of the Az derivative, the spectral change was small, as observed in Figure 5a; however, in the magnified spectra shown in Figure 5b, an absorption enhancement of $\sim 360 \mathrm{~nm}$ is observed for the $\pi \pi^{\star}$ band of Az. The absorbance increment at $360 \mathrm{~nm}$ was $\sim 0.005$, indicating that the Az derivative was attached at a sub-monolayer level. In comparison with the absorbance of a monolayered Langmuir-Blodgett film $(\sim 0.02),{ }^{9,10}$ the attached amount was approximately one-fourth of a well-packed monolayer.

Figure 6 displays the photoresponse of the nematic LC (5CB) cell prepared with two Az-modified polyimide substrates. In crossed polarizers, the entire area was dark in the trans-Az state due to the

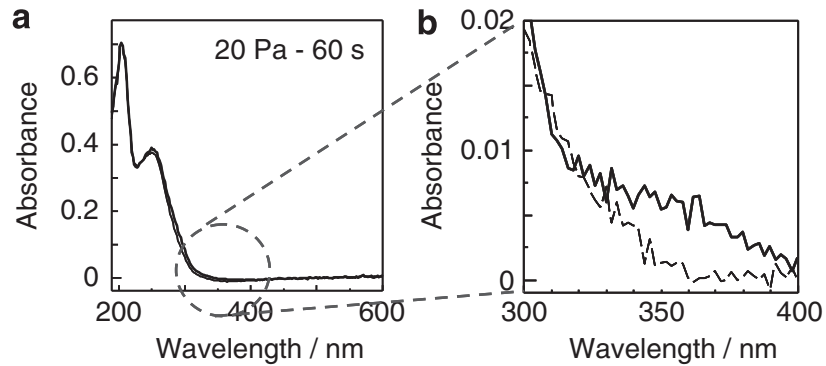

Figure 5 UV-visible absorption spectra of a polyimide film without modification (dashed line) and after modification with $5 \mathrm{Az} 10 \mathrm{COCl}$ (solid line). In (a) and (b), the complete spectra and magnified portions are presented, respectively.

homeotropic alignment of the LC. When a spot UV light was irradiated, the exposed area became bright, and a Schlieren texture was observed. This result demonstrates that the nematic LC was randomly oriented on the cis-Az surface. Such behavior is typically observed in an LC cell with Az-modified substrates by S-adenosylmethionine ${ }^{1,7,8}$ or a Langmuir-Blodgett monolayer., ${ }^{9,10}$ Although the attached $\mathrm{Az}$ derivative was sparse as mentioned above, the surface possessed sufficient ability for photoswitching to the zenithal direction. The present photochemical approach provides a photoresponsive command surface equivalent to that prepared using conventional methods. ${ }^{1,7-10}$

Attachment of cinnamate unit and alignment control of LC cells We then attempted to introduce a photocrosslinkable cinnamoyl group onto the polyimide surface. Two cinnamoyl units undergo 


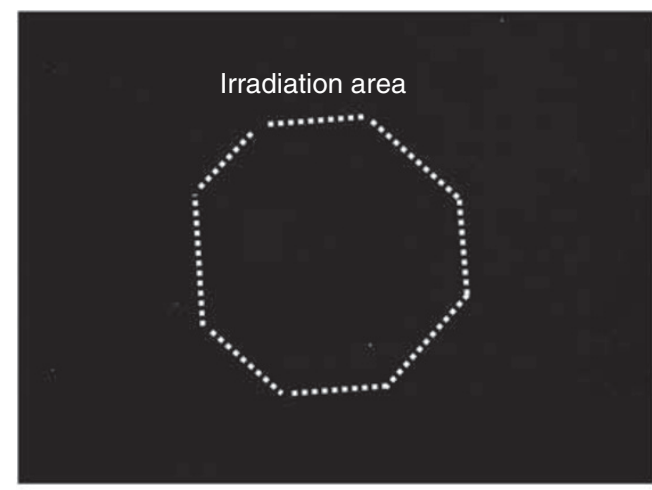

$$
\operatorname{UV}(365 \mathrm{~nm}) \downarrow_{\downarrow} \mid \text { Vis }(436 \mathrm{~nm})
$$

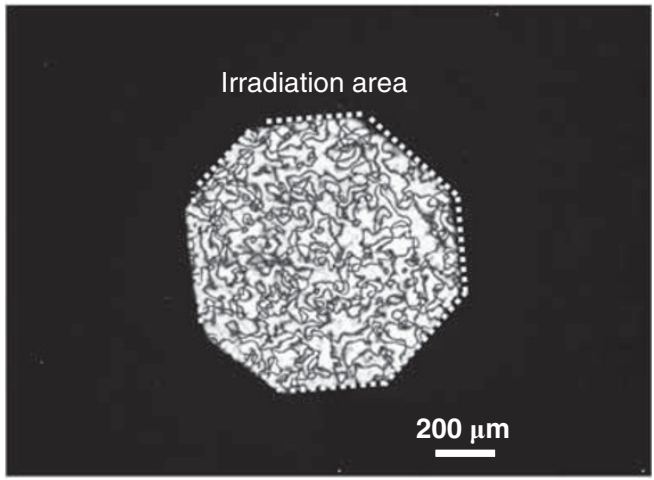

Figure 6 Out-of-plane orientation switching of 5CB in an Az-modified LC cell. UV $(365 \mathrm{~nm})$ and visible $(436 \mathrm{~nm})$ light was irradiated alternately on the spot area in the center.

$(2+2)$ photodimerization to form a cyclobutane ring. Figure $7 \mathrm{a}$ presents the UV-visible absorption spectra of the polyimide film modified with cinnamoyl chloride before and after VUV light irradiation. For the non-irradiated film, the absorption band corresponding to the cinnamoyl group was hardly observed (Figure 7a, dashed line). However, the VUV-irradiated film exhibited a large absorption band (absorbance $>0.03$ ) peaking at $295 \mathrm{~nm}$, ascribed to the attachment of the cinnamate group. The larger absorption band represents the greater attachment of the cinnamate units than $\mathrm{Az}$ units on the polyimide surface. As the molar extinction coefficient of $\mathrm{Az}$ and the cinnamoyl units are comparable, the attached amount of the cinnamate unit was 6-7 times greater than that of the $\mathrm{Az}$ derivative. Steric bulkiness is assumed to affect the reactivity. The smaller cinnamic acid chloride appears to react more readily with the surface. However, its amount is still too small, indicating a molecular layer formation whose coverage is below a bilayer level.

To induce angular-selective photoreaction, $313 \mathrm{~nm}$ LPL was irradiated on the cinnamate-modified film. Figure $7 \mathrm{~b}$ depicts the polarized UV-vis absorption spectra of the films before and after LPL irradiation. The in-plane selective photoreaction was evaluated by measuring the absorbance of the $\pi-\pi^{\star}$ band of the cinnamate group obtained using polarized absorption spectroscopy. Here, $A_{/ /}$and $A_{\perp}$ denote the spectra obtained by probing a polarized light beam parallel or perpendicular, respectively, to the direction of the actinic $313 \mathrm{~nm}$ LPL. After LPL irradiation, the absorption band near $295 \mathrm{~nm}$ slightly
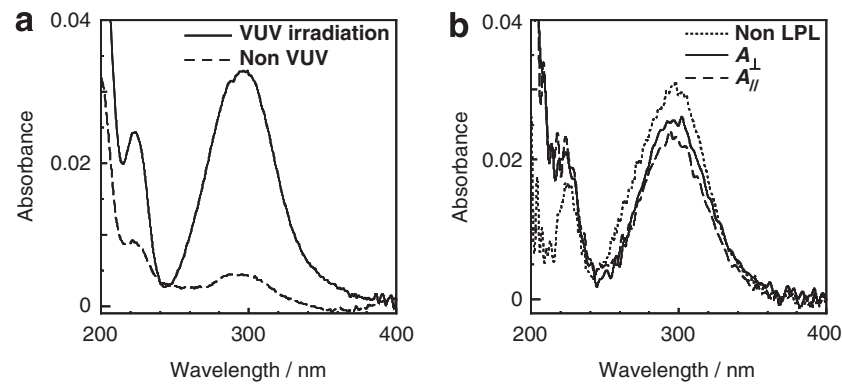

Figure 7 (a) UV-vis absorption spectra of a modified polyimide film reacted with cinnamoyl chloride without (dashed line) and after VUV light irradiation at $20 \mathrm{~Pa}$ for $60 \mathrm{~s}$. (b) Polarized UV-vis absorption spectra of the modified polyimide film with cinnamoyl chloride before (non-LPL) and after LPL irradiation at $313 \mathrm{~nm}$.
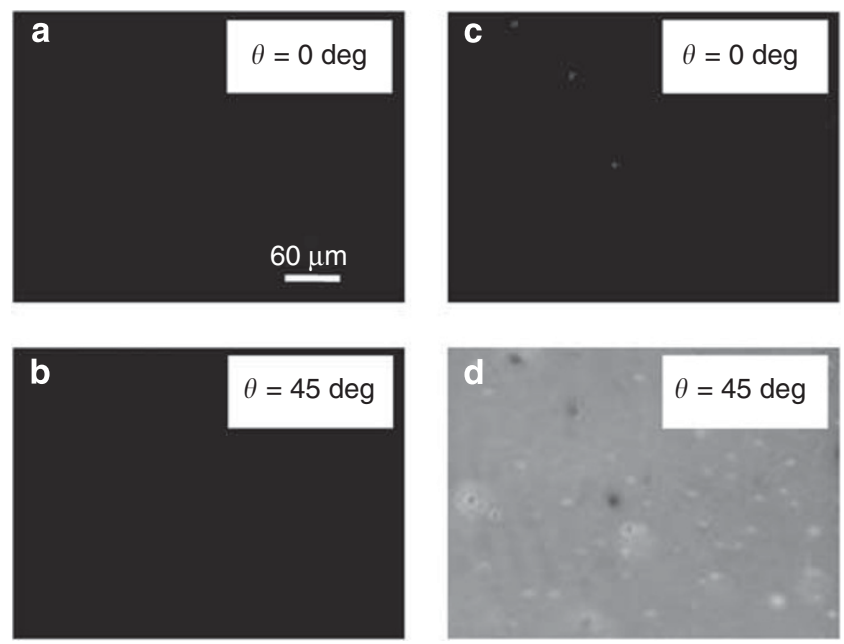

Figure $\mathbf{8}$ Optical microscopic images of the vertical alignment LC cell observed through the crossed polarizers at the initial state, (a) and (b), and after application of a 4-V electric field, (c) and (d). The value of $\theta$ is the angle between the polarizer and the direction of LPL.

decreased, and $A_{/ /}$became lower than $A_{\perp}$, suggesting that the cinnamoyl group underwent anisotropic selective photodimerization to form a cyclobutane ring.

We prepared the LPL-irradiated films of the polyimide on an indium-tin-oxide substrate using the same procedure, and DON-103 was sandwiched between them. Figures $8 \mathrm{a}$ and $\mathrm{b}$ present the optical microscopic images of the LC cell fabricated with the two cinnamate photoalignment layers before application of an electric field. Regardless of the angle $\theta$ between the LPL and the crossed polarizers, only dark-field images were observed. This fact indicates that the nematic LC adopts homeotropic alignment to the substrate plane in contrast to poly(vinyl cinnamate) ${ }^{3}$ or other series of cinnamate-containing polymer films, ${ }^{37,38}$ where parallel homogeneous (uniaxial) alignment is formed after LPL irradiation. The present polyimide film possesses cholesteryl side chains in the original state, and these long-chained moieties may induce the homeotropic alignment in the initial state. After modification with the cinnamoyl group, the nematic LC adopts the homeotropic alignment. As argued in the text, this fact clearly indicates that the cholesteryl group, a homeotropically aligning unit, remains. This assumption is in agreement with the contact angle measurements, where the contact angle of water was reduced by VUV 


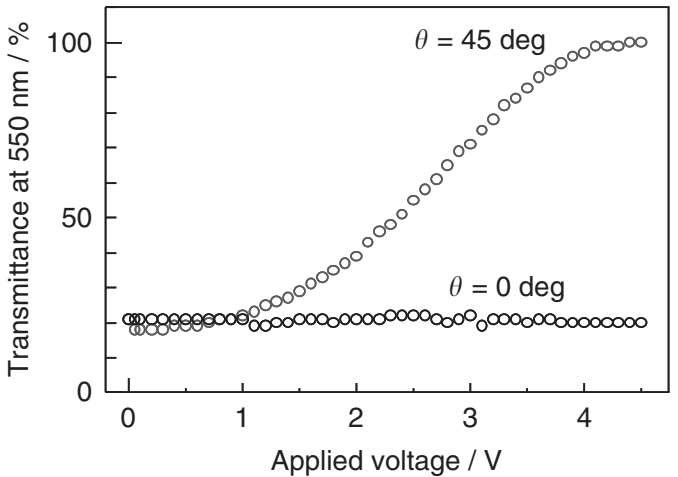

Figure 9 Intensity of transmitted light at $550 \mathrm{~nm}$ as a function of the applied voltage for the LC cell fabricated with a cinnamate-modified polyimide film. $\theta$ is the same as described in Figure 8. $T=0 \%$ was measured under a crossed polarizer without the LC cell.

irradiation and saturated at $75^{\circ}$. The saturated value exhibits a more hydrophobic nature than expected for a fully oxidized surface. Some hydrocarbon parts, possibly cholesteryl units, should remain on the surface.

By applying an electric field of $4 \mathrm{~V}$ at $500 \mathrm{~Hz}$, however, the cell exhibited a bright-field image at $\theta=45^{\circ}$, and the bright- and darkfield imagees were inverted for every $45^{\circ}$ rotation thereafter (Figures $8 \mathrm{c}$ and $\mathrm{d}$ ). This electro-optical behavior indicates that the axialselective photoreaction of the cinnamate group at the surface induced the homogeneous alignment of DON-103 with negative dielectric anisotropy. In the homogeneous alignment, the nematic director (long axis of the LC molecules) is aligned orthogonal to the electric field of the LPL, as confirmed by the polarized absorption spectroscopic data of the LC cell containing a dichroic dye (LCD-118, $0.1 \%$ by weight). In the present cell, the photoinduced anisotropy of the modified polyimide exhibited high thermal stability. The homogeneous alignment was retained even after heating $>120^{\circ} \mathrm{C}$, which agreed with the results obtained by the spin-cast films of poly(vinyl cinnamate) and other cinnamate-containing polymers ${ }^{37,38}$ after photocrosslinking.

The vertical alignment-LC cell performance upon application of an electric field was further investigated. The transmittance of the cell was measured. Figure 9 illustrates the transmitted light intensity of the cell at $550 \mathrm{~nm}$ under crossed polarizers as a function of the applied voltage. Here, the zero transmittance level $(T=0 \%)$ was obtained by a measurement without the LC cell. As the applied voltage increased, the transmitted light intensity increased at $\theta=45^{\circ}$, whereas that at $\theta=0^{\circ}$ did not change under the same conditions. In this manner, the resulting photoalignment layer provides a vertical alignment LC device.

\section{CONCLUSION}

The surface of a polyimide film with a cholesteryl side moiety was selectively oxidized by VUV $(172 \mathrm{~nm})$ treatment without changing the inner chemical structure of the polyimide film. On the oxidized surface, chemical functional groups, such as hydroxyl groups, are generated and can be subjected to the formation of an ester bond using select photofunctional acid chlorides. This newly proposed photochemical procedure is simple but should be widely applicable to many types of polymer surfaces. As observed in this work, this method leads to the facile introduction of both photoisomerizable $\mathrm{Az}$ and photocrosslinkable cinnamate units in the same procedure, suggesting the versatility of the process. The present process is an alternative to the conventional SAM formation or the LangmuirBlodgett method. Moreover, it should be stressed that the surface nature overlaps with the characteristics of the bulk polymer existing underneath, as indicated for the case of the cinnamoyl unit. For this reason, we expect that a more compositive and tunable functional surface can be produced using this approach.

\section{ACKNOWLEDGEMENTS}

We thank Mr J Isayama for helpful discussions. This work was supported by a Grant-in-Aid for Scientific Research (in Priority Areas 'New Frontiers in Photochromism (no. 471), and Basic Research (S) (23225003)) from the Ministry of Education, Culture, Sports, Science and Technology (MEXT) of Japan and the JST-PRESTO program in Japan.

1 Ichimura, K., Suzuki, Y., Seki, T., Hosoki, A. \& Aoki, K. Reversible change in alignment mode of nematic liquid crystals regulated photochemically by 'command surfaces' modified with an azobenzene monolayer. Langmuir 4, 1214-1216 (1988).

2 Gibbons, W. M., Shannon, P. J., Sun, S. T. \& Swetlin, B. J. Surface-mediated alignment of nematic liquid crystals with polarized laser light. Nature 351, 49-50 (1991).

3 Schadt, M., Schmitt, K., Kozinkov, V. \& Chigrinov, V. Surface-induced parallel alignment of liquid crystals by linearly polymerized photopolymers. Jpn. J. Appl. Phys 31, 2155-2164 (1992)

4 Ichimura, K. Photoaligment of liquid crystal systems. Chem. Rev. 100, 1847-1873 (2000).

5 O'Neill, M. \& Kelly, S. K. Induced surface alignment for liquid crystal displays. J. Phys. D: Appl. Phys 33, R67-R84 (2000).

6 Yaroshchuk, O. \& Reznikov, Y. Photoalignment of liquid crystals: basics and current trends. J. Mater. Chem. 22, 286-300 (2012).

7 Aoki, K., Tamaki, T., Seki, T., Kawanishi, Y. \& Ichimura, K. Regulation of alignment of cyanobiphenyl liquid crystals by azobenzene molecular films. Langmuir 8, 1014-1017 (1992).

8 Aoki, K., Seki, T., Suzuki, Y., Tamaki, T., Hosoki, A. \& Ichimura, K. Factors affecting photoinduced alignment regulation of cyclohexanecarboxylate type nematic liquid crystals by azobenzene molecular films. Langmuir 8, 1007-1013 (1992).

9 Seki, T., Sakuragi, M., Kawanishi, Y., Tamaki, T., Fukuda, R. \& Ichimura, K. 'Command surfaces' of Langmuir-Blodgett films. Photoregulation of liquid crystal alignment by molecularly tailored surface azobenzene layers. Langmuir 9, 211-218 (1993).

10 Seki, T., Fukuda, R., Sakuragi, M., Kawanishi, Y., Suzuki, Y., Tamaki, T. \& Ichimura, K. Modulated photoregulation of liquid crystal alignment by azobenzene LangmuirBlodgett layers. Thin Solid Films 210/211, 836-838 (1992).

11 Idage, S. B. \& Badrinarayanan, S. Surface modification of polystyrene using nitrogen plasma. An X-ray photoelectron spectroscopy study. Langmuir 14, 2780-2785 (1998).

12 France, R.M. \& Short, R.D. Plasma treatment of polymers: the effects of energy transfer from an argon plasma on the surface chemistry of polystyrene, and polypropylene. A high-energy resolution X-ray photoelectron spectroscopy study. Langmuir 14, 4827-4835 (1998).

13 Dupont-Gillain, Ch. C., Adriaensen, Y., Derclaye, S. \& Rouxhet, P.G. Plasma-oxidized polystyrene: wetting properties and surface reconstruction. Langmuir 16, 8194-8200 (2000).

14 Schulz, U., Munzert, P. \& Kaiser, N. Surface modification of PMMA by DC glow discharge and microwave plasma treatment for the improvement of coating adhesion. Surf. Coat. Technol 142, 507-511 (2001).

15 Pascu, M., Vasile, C. \& Gheorghiu, M. Modification of polymer blend properties by argon plasma/electron beam treatment: surface properties. Mater. Chem. Phys. 80, 548-554 (2003)

16 Zhang, D., Dougal, S.M. \& Yeganeh, M.S. Effects of UV irradiation and plasma treatment on a polystyrene surface studied by IR -visible sum frequency generation spectroscopy. Langmuir 16, 4528-4532 (2000).

17 Onyiriuka, E.C., Hersh, L.S. \& Hertl, W. Solubilization of corona discharge- and plasma-treated polystyrene. J. Colloid Interface Sci. 144, 98-102 (1991).

18 Gassan, J. \& Gutowski, V. S. Effects of corona discharge and UV treatment on the properties of jute-fibre epoxy composites. Compos. Sci. Technol 60, 2857-2863 (2000).

19 Friedrich, J., Wigant, L., Unger, W., Lippitz, A. \& Wittrich, H. Corona, spark and combined UV and ozone modification of polymer films WeBP23. Surf. Coat.Technol 98, 879-885 (1998).

20 Teare, D. O. H., Emmison, N., Ton-That, C. \& Bradley, R.H. Cellular attachment to ultraviolet ozone modified polystyrene surfaces. Langmuir 16, 2818-2824 (2000).

21 Dong, H. \& Bell, T. State-of-the-art overview: ion beam surface modification of polymers towards improving tribological properties. Surf. Coat. Technol 111, 29-40 (1999).

22 Netcheva, S. \& Bertrand, P. Surface topography development of thin polystyrene films under low energy ion irradiation. Nucl. Instrum. Methods Phys. Res. B 151, 129-134 (1999).

23 Iwaki, M. Ion surface treatments on organic materials. Nucl. Instrum. Methods Phys. Res. B 175-177, 368-374 (2001). 
24 Onyiriuka, E.C. The effects of high-energy radiation on the surface chemistry of polystyrene: a mechanistic study. J. Appl. Polym. Sci 47, 2187-2194 (1993).

25 Marmey, P., Porté, M.C. \& Baquey, Ch. PVDF multifilament yarns grafted with polystyrene induced by $\gamma$-irradition: Influence of the grafting parameters on the mechanical properties. Nucl. Instrum. Methods Phys. Res. B 208, 429-433 (2003).

26 Astumian, R.D. \& Schelly, Z.A Geometric effects of reduction of dimensionality in interfacial reactions. J. Am. Chem. Soc. 106, 304-308 (1984).

27 Fadeev, A.Y. \& McCarthy, T. J. Surface modification of poly(ethylene terephthalate) to prepare surfaces with silica-like reactivity. Langmuir 14, 5586-5593 (1998).

28 Chen, W. \& McCarthy, T. J. Chemical surface modification of poly(ethylene terephthalate). Macromolecules 31, 3648-3655 (1998).

29 Hozumi, A., Inagaki, H. \& Kameyama, T. The hydrophilization of polystyrene substrates by 172-nm vacuum ultraviolet light. J. Colloid Interface Sci $\mathbf{2 7 8}$, 383-392 (2004).

30 Hozumi, A., Masuda, T., Hayashi, K., Sugimura, H., Takai, O. \& Kameyama, T. Spatially defined surface modification of poly(methyl methacrylate) using $172 \mathrm{~nm}$ vacuum ultraviolet light. Langmuir 18, 9022-9027 (2002).

31 Roland, R. P., Bolle, M. \& Anderson, R. W. Low temperature photochemical vapor deposition of $\mathrm{SiO}_{2}$ using $172 \mathrm{~nm} \mathrm{Xe}{ }_{2}{ }^{*}$ excimer lamp radiation with three oxidant chemistries: $\mathrm{O}_{2}, \mathrm{H}_{2} \mathrm{O} / \mathrm{O}_{2}$, and $\mathrm{H}_{2} \mathrm{O}_{2}$. Chem. Mater 13, 2493-2500 (2001).
32 Hong, L., Sugimura, H., Furukawa, T. \& Takai, O. Photoreactivity of alkylsilane self-assembled monolayers on silicon surfaces and its application to preparing micropatterned ternary monolayers. Langmuir 19, 1966-1969 (2003).

33 Tsuji, M., Kawahara, T., Kamo, N. \& Miyano, M. Photochemical removal of benzene using $172 \mathrm{~nm} \mathrm{Xe} 2$ excimer lamp in $\mathrm{N}_{2} / \mathrm{O}_{2}$ mixtures at atmospheric pressure. Bul. Chem. Soc. Jpn. 83, 582-591 (2010)

34 Lee, K.-W., Park, S.-H., Lien, A., Durning, C. \& Fukuro, H. Microscopic molecular reorientation of alignment layer polymer surfaces induced by rubbing and its effects on LC pretilt angles. Macromolecules 29, 8894-8899 (1996).

35 Miyata, F., Nemoto, N., Nagase, Y., Abe, J., Hasegawa, M. \& Shirai, Y. Polyamides alternately substituted with an aliphatic cyclic moiety and an NLO-phore for secondorder nonlinear optics. Macromol. Chem. Phys. 199, 1465-1471 (1998).

36 Okui, K. \& Ito, A. Thickness determination of thin oxide layers on metal surfaces using X-ray photoelectron spectroscopy. Bunseki Kagaku 40, 691-696 (1991).

37 Ichimura, K., Akita, Y., Akiyama, H., Hayashi, Y. \& Kudo, K. Role of E/Z photoisomerization of cinnamate side chains attached to polymer backbones in the alignment photoregulation of nematic liquid crystals. Jpn. J. Appl. Phys 35, L992-L995 (1996).

38 Ichimura, K., Akita, Y., Akiyama, H., Kudo, K. \& Hayashi, Y. Photoreactivity of polymers with regioisomeric cinnamate side chains and their ability to regulate liquid crystal alignment. Macromolecules 30, 903-911 (1997). 